\title{
Diffuse interstellar bands in the Local Group: From the Milky Way, the Magellanic Clouds to the Andromeda galaxy
}

\author{
N. L. J. $\operatorname{Cox}^{1}$ and M. A. Cordiner ${ }^{2}$ \\ ${ }^{1}$ Herschel Science Centre, European Space Astronomy Centre, European Space Agency, \\ Villanueva de Cañada, Madrid, Spain; email: nick.cox@sciops.esa.int \\ ${ }^{2}$ Astrophysics Research Centre, School of Mathematics and Physics, Queen's University, \\ Belfast, U.K
}

\begin{abstract}
We report on recent developments in the study of diffuse interstellar bands in the Local Group galaxies. We present preliminary results on the detection of the $5780 \AA$ DIB toward 17 targets in the vicinity of NGC 206 in M31.
\end{abstract}

Keywords. Local Group, ISM: lines and bands, ISM: molecules

\section{Diffuse interstellar bands}

The diffuse interstellar bands (DIBs) constitute a group of over 200 optical interstellar (IS) absorption features observed toward reddened stars (see review by Herbig 1995). For Galactic lines-of-sight their equivalent widths correlate with the amount of interstellar reddening, $E_{B-V}$, as well as with the Na I column density (e.g., Herbig 1993). To this day it is still debated whether they arise from the dust or the gas component of the ISM. However, although not one of the DIBs has been identified, there is strong evidence that the carriers are composed of organic material (Sarre 2008). The substructure present in many of the DIB profiles indicate that the carriers are large gas-phase molecules (Sarre 1995, Ehrenfreund \& Foing 1996). Likely candidates include UV-resistant organics such as PAHs, fullerenes and carbon chains (see, e.g., Salama 1996). Unfortunately it is not yet possible to compare directly the observational spectra with laboratory spectra of large ionised PAHs in the gas-phase (Salama 2008).

In the last decade, observations of diffuse interstellar bands (DIBs) have provided important insights into the chemical characteristics of possible carrier compounds (Sarre 2006). Recent observations show a strong dependence of DIB strength on the local environment in terms of cloud density and exposure to the interstellar radiation field (Cox \& Spaans 2006). In particular, extragalactic environments have contributed recently to our knowledge of DIB behavior (Cox et al. 2006, 2007).

Our goal is to examine the link between the physical and chemical conditions in the ISM and shed light on the nature of the large molecules and very small grains believed to give rise to the DIBs.

\section{The Local Group diffuse ISM}

The Local Group is an ideal laboratory for detailed studies of the ISM over a broad range of physical and chemical conditions. Previous research of DIBs in our nearest neighbouring galaxies has focused on the Large and Small Magellanic Clouds (e.g., Ehrenfreund 
et al. 2002, Cox et al. 2006, 2007, Welty et al. 2006) where the effects on DIB strengths of the higher gas-to-dust ratios, lower metallicities, lower $R_{V}$ and higher interstellar radiation fields were examined. Beyond the Magellanic Clouds, studies are sparse, confined to sightlines probed by sufficiently bright supernovae (e.g., Sollerman et al. 2005, Cox \& Patat 2008) or background quasars (e.g., York et al. 2006, Ellison et al. 2008). Cordiner et al. (2008) detected for the first time DIBs in the spectra of two bright stars in M31, the Andromeda galaxy. In the next section we present new preliminary results on the 5780 DIB detection in 15 additional M31 sightlines.

The LMC, SMC and M31 have different global properties of the ISM. The Large Magellanic Cloud (LMC) and the Small Magellanic Cloud (SMC) have metallicities that are smaller by factor 4 and 10, respectively, with respect to the Milky Way. M31 has a metallicity close to solar (e.g., Urbaneja et al. 2005). A similar trend can be seen for the dust-to-gas ratio. Also their dust extinction curves are very different. The dust in the Magellanic Clouds contains more smaller dust particles (stronger FUV extinction) than the MW. The strength of the UV radiation field is also stronger in the LMC and SMC. The M31 galaxy, on the other hand shows a low interstellar UV flux (Pagani 1999) accordingly, the M31 ISM has been subject to relatively little UV-processing which may also explain the anomalous mid-IR spectrum. Xu \& Helou (1994) find a lack of small graphitic dust grains in the ISM of M31.

\section{New developments: low-resolution spectra of M31 (super)giants}

In the study by Cordiner et al. (2008), low-resolution $(R \approx 3300)$, optical spectra of 72 early-to-mid-type giants and supergiants in the vicinity of the M31 OB78 association (NGC206, Van den Bergh 1964) were obtained with the Keck DEIMOS spectrograph. Five of the strongest narrow DIBs, $\lambda \lambda$ 5780, 5797, 6203, 6283 and $6613 \AA$, were detected in the ISM of M31. The exposure of the red spectra was 1.5 hours during excellent conditions, with seeing $0.5-0.8^{\prime \prime} .17$ targets were selected from this set of spectra. These spectra, with highest $\mathrm{S} / \mathrm{N}$ and earliest spectral-type (less stellar lines), showed evidence of absorption consistent with the 5780 DIB. The radial velocities, between -530 and $590 \mathrm{~km} \mathrm{~s}^{-1}$, of the interstellar gas towards these targets was derived from the the $\mathrm{Na}$ I doublet, and subsequently used to fit synthetic DIB profiles to the observed spectra. The spectra of the selected sightlines and the profile fits are shown in figure 1 . Note that these spectra also include MAG 63885 and MAG 70817 from the study by Cordiner et al. (2008).

In figure 2 the observed DIB strengths are compared with the interstellar sodium column density and the interstellar reddening, respectively. For comparison the Galactic relationships are also shown. Due to the low spectral resolution there is likely to be contamination of the NaI column densities due to stellar sodium, particularly for the later spectral types. The uncertainties in the stellar photometry and spectral types make an accurate determination of the reddening also difficult.

With these possible sources of error in mind, it is still clear from Figure 2 that the DIBs so far detected in M31 are, on average, stronger than Galactic DIBs relative to $E_{B-V}$. The ISM in the vicinity of OB78 evidently provides environments which are highly favourable for the production of DIB carriers but may have join up atypical properties compared to the rest of M31 due to the abundance of early-type stars compared with the rest of M31. The spectrum of DIBs observed in the vicinity of OB78 in M31, where the metallicity is approximately equal to solar, is found to be comparable to (or stronger than) that observed locally in the ISM of the Milky Way. 

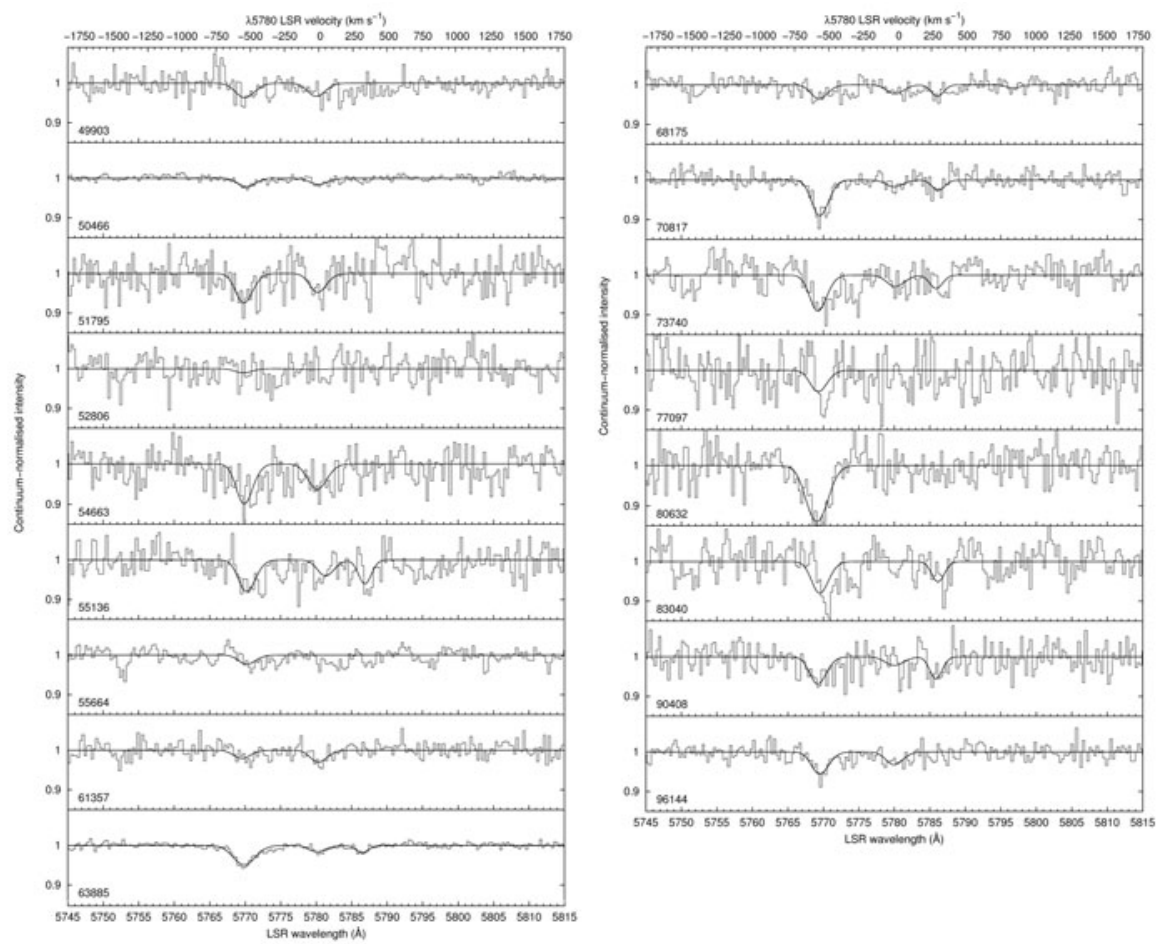

Figure 1. The $\lambda 5780$ (left) and $\lambda 6283$ (right) DIB spectral ranges of 17 targets in the OB78 (NGC206) association in the southern outskirts of M31. Target numbers are from Magnier 1992. The 5780 DIB is detected in 17 sightlines and the 6283 DIB in 7 sightlines.
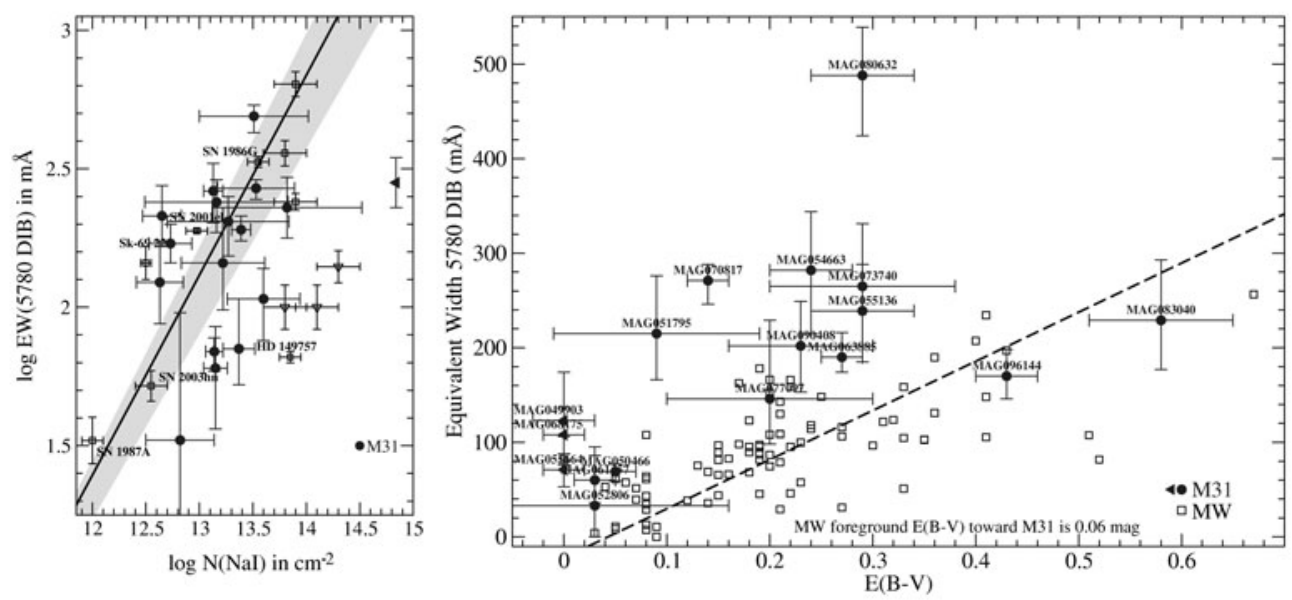

Figure 2. Correlation of $\lambda 5780$ DIB equivalent widths with neutral sodium column density (left) and reddening (right). The linear fits represent the Galactic behaviour (see, e.g., Herbig 1993). The small dots in the right panel are from a Galactic DIB study (Vos et al. 2008).

\section{Progress and future work}

The low resolution of the spectra presented precludes a detailed analysis of the structure and composition of the M31 ISM. Nevertheless, the results here (preliminary) and 
in Cordiner et al. (2008) verify the feasibility of studying in more detail the atoms, molecules and diffuse bands in the ISM of the Local Group members M31 and M33. The next step will be to obtain high-resolution, high signal-to-noise spectra in order to consolidate these results and to identify whether the unusually strong DIBs observed are a common feature of M31 or are unique to the OB78 region. The chemical properties of the sightlines studied in this article may be further elucidated by examining the nature of the dust, particularly the UV extinction, which will be possible with the new 'COS' and the refurbished 'STIS' instruments on the HST.

\section{References}

Cordiner, M. A., Cox, N. L. J., Trundle, C., Evans, C. J., Hunter, I., Przybilla, N., Bresolin, F., \& Salama, F. 2008, A\&AA, 480, L13

Cox, N. L. J., Cordiner, M. A., Cami, J., Foing, B. H., Sarre, P. J., Kaper, L., \& Ehrenfreund, P. 2006, A\& $A, 447,991$

Cox, N. L. J., Cordiner, M. A., Ehrenfreund, P., Kaper, L., Sarre, P. J., J., Foing, B. H., Spaans, M., Cami, J., Sofia, U. J., Clayton, G. C., Gordon, K. D., \& Salama, F. 2007, A\&3A, 470, 941

Cox, N. L. J. \& Patat, F. 2008, A\&A, accepted

Cox, N. L. J. \& Spaans, M. 2006, A\& A, 451, 973

Ehrenfreund, P. \& Foing, B. H. 1996, A\&A, 307, L25.

Ehrenfreund, P., Cami, J., Giminez-Vicente, J., et al. 2002, ApJ, 576, L117

Ellison, S. L., York, B. A., Murphy, M. T., Zych, B. J., Smith, A. M., \& Sarre, P. J. 2008, MNRAS (Letters), 383, L30

Herbig, G. H. 1993, ApJ, 407, 142

Herbig, G. H. 1995, ARA\&A, 33, 19

Magnier, E. A., Lewin, W. H. G., van Paradijs, J., Hasinger, G., Jain, A., Pietsch, W., \& Truemper, J. 1992, A\&SAS, 96, 379

Pagani, L., Lequeux, J., Cesarsky, D., Donas, J., Milliard, B., Loinard, L., \& Sauvage, M. 1999, $A \mathscr{E} A, 351,447$

Salama, F. 2008, this volume

Salama, F., Bakes, E. L. O., Allamandola, L. J., \& Tielens, A. G. G. M. 1996, ApJ, 458, 621

Sarre, P. J. 2006, Journal of Molecular Spectroscopy, 238, 1

Sarre, P. J. 2008, this volume

Sarre, P. J., Miles, J. R., Kerr, T. H., Hibbins, R. E., Fossey, S. J., \& Somerville, W. B. 1995, MNRAS, 277, L41

Sollerman, J., Cox, N. Mattila, S. Ehrenfreund, P., Kaper, L., Leibundgut, B., \& Lundqvist, P. 2005, A\&A, 429, 559.

Urbaneja, M. A., Herrero, A., Kudritzki, R. P., Najarro, F., Smartt, S. J., Puls, J., Lennon, D. J., \& Corral, L. J. 2005, ApJ, 635, 311

Van den Bergh, S. 1964, ApJS, 9, 65

Vos, D. A. I., Cox, N. L. J., Kaper, L., \& Ehrenfreund, P. 2008, A\& A, submitted

Welty, D. E., Federman, S. R., Gredel, R., Thorburn, J. A., \& Lambert, D. L. 2006, ApJS, 165, 138

Xu, C. \& Helou, G., 1994, ApJ, 426, 109

York, B. A., Ellison, S. L., Lawton, B., Churchill, C. W., Snow, T. P., Johnson, R. A., \& Ryan, S. G. 2006, ApJ, 647, L29

\section{Discussion}

IRVINE: A this point, what is the most distant Galaxy in which you have seen DIBs?

Cox: We have detected narrow DIBs in NGC1448 toward a supernova, and also recently in M100 (also toward a supernova), which is a little bit closer. Also two narrow DIBs have been detected in a DLA system at $z \sim 0.5$ (work by York, Ellison, Lawton, Snow and collaborators). 